\title{
Culture in vitro d'ovules et d'embryons de vigne (Vitis vinifera L.) appliquée à la sélection de variétés de raisins de table sans pépins
}

\author{
A. Bouquet ${ }^{1}$ et H. P. Davis 2 \\ avec la collaboration technique d'Y. Danglot ${ }^{1}$ et de C. Rennes ${ }^{3}$ \\ 1 INRA, Station de recherches viticoles, domaine du Chapitre, 34750 Villeneuve-lès-Maguelonne, France; \\ 2 CSIRO, Division of Horticultural research, Merbein, Victoria 3505, Australie; \\ 3 INRA, Station de recherches viticoles, domaine de Vassal, 34340 Marseillan, France
}

(reçu le 23 janvier 1989, accepté le 5 avril 1989)

Résumé - Les méthodes conventionnelles de création de variétés de raisins de table sans pépins (apyrènes) font appel à des croisements nécessitant l'utilisation de variétés classiques à pépins comme géniteurs femelles. Mais la faible proportion de plants sans pépins dans les descendances ainsi obtenues et l'expression souvent imparfaite du caractère apyrène limitent considérablement les possibilités et l'efficacité de la sélection. La technique du sauvetage d'embryons par culture d'ovules in vitro permet d'obtenir des descendances à partir de croisements entre variétés apyrènes. Cette technique, appliquée en 1987-1988 à 15610 ovules fécondés issus de 21 croisements différents, a permis d'obtenir plus de 1200 plantes, et de disposer ainsi d'une variabilité génétique considérable pour les travaux de sélection de nouvelles variétés de raisins de table capables de satisfaire les exigences des producteurs et d'offrir un produit nouveau et attractif aux consommateurs.

amélioration génétique - croisements intraspécifiques - sténospermocarpie - polyembryonie - qualité du fruit

Summary - In ovulo and in vitro embryo culture for breeding seedless table grapes (Vitis vinifera L.). Conventional breeding of seedless grapes involves hybridization between seeded cultivars used as females and seedless varieties used as pollinators. But the low proportion of seedless plants in the progenies and the expression often imperfect of the seedless character limit considerably the possibilities and the efficiency of the selection. In ovulo and in vitro embryo rescue allows recovery of progenies from abortive ovules of seedless $x$ seedless crosses. This technique, applied in 1987-1988 to 15610 ovules from 21 different crosses, gave more than 1200 plantlets that represents a considerable genetic variability for the selection of new table grape varieties able to satisfy the needs of the producers and to offer a new and attractive product to the consumers.

genetic improvement - intraspecific hybridization - stenospermocarpy - polyembryony - fruit quality

\section{Introduction}

La création de variétés de raisins de table sans pépins est un axe de recherches important dans les programmes d'amélioration de la vigne développés en France et dans de nombreux pays. En effet, ce type de variétés, qui est utilisé depuis longtemps pour la production de raisins secs et de raisins de conserve, est de plus en plus apprécié des consommateurs de fruits frais dans les pays anglo-saxons (Etats-Unis, Australie...) et susceptible de relancer la consommation et la production de raisins de table en France.
Les variétés sans pépins, également appelées apyrènes, utilisables pour la production de raisins de table, sont de type sténospermocarpique (Stout, 1936). La variété la plus connue et la plus cultivée dans le monde est la Sultanine blanche, également appelée Thompson seedless en Californie. En fait, les variétés de ce type ne sont pas des variétés sans pépins au sens strict, comme le sont par exemple les variétés parthénocarpiques tel le Corinthe noir, variété grecque dont la taille extrêmement réduite des baies en limite l'utilisation à la seule production de raisins secs. Dans le cas de la Sultanine blanche et des varié- 
tés qui en sont dérivées, il y a fécondation, mais le développement de l'embryon et/ou de l'endosperme est incomplet et s'interrompt quelques semaines après la floraison (Nitsch et al., 1960; Barritt, 1970). Les baies possèdent donc des ébauches de pépins rudimentaires, de taille très réduite (Fig. 1), généralement mous et dépourvus de téguments lignifiés, dont la perception par le consommateur est limitée, voir nulle. La dimension moyenne des baies est naturellement inférieure à celle des baies de variétés à pépins, mais peut être augmentée par des traitements à l'acide gibberellique (Mavrikios, 1977) ou par la sélection génétique.

Les méthodes conventionnelles d'amélioration reposent sur des croisements entre variétés à pépins, utilisées comme parents femelles après suppression des étamines avant la floraison, et variétés sans pépins utilisées comme pollinisateurs. Mais la proportion de plantes apyrènes dans les descendances ainsi obtenues est faible et dépasse rarement 10 à 15\% (Weinberger et Harmon, 1964; Loomis et Weinberger, 1979). De plus, l'expression du caractère apyrène est souvent imparfaite, avec présence dans certaines baies d'ébauches de pépins nettement perceptibles, quelquefois lignifiées, qui nuisent à la qualité commerciale des fruits.

Les perspectives d'un réel progrès génétique dans ce domaine reposent donc sur la possibilité d'obtenir des descendances sexuées à partir de croisements entre variétés apyrènes, utilisées simultanément comme parents mâles et femelles, en assurant grâce à la culture in vitro le développement complet des embryons issus de ces croisements.

La culture in vitro d'embryons n'est pas à proprement parler une technique nouvelle et originale, puisque les premiers travaux dans ce domaine datent du début du siècle (Hannig, 1904). Appliquée à de nombreuses espèces, elle s'est révélée être un outil extrêmement utile, sinon

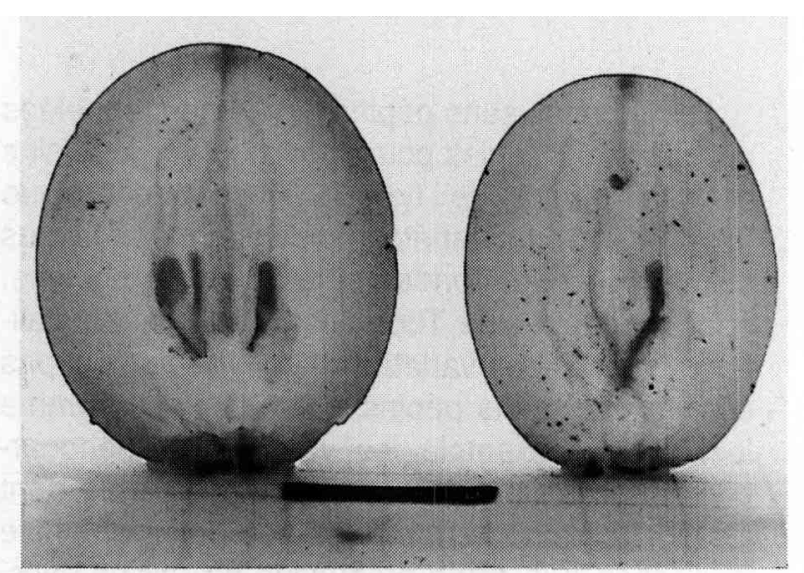

Fig. 1. Ebauches de pépins à l'intérieur d'une baie de la variété Canner seedless (grossissement : $\times 3$ ). indispensable, dans le cas d'hybridations rendues difficiles soit par l'éloignement génétique des espèces impliquées (Islam, 1964), soit par des niveaux différents de ploïdie (Stewart et Hsu, 1978). Elle a, de même, été couramment utilisée pour la production de plantes haploïdes, chez l'orge notamment (Kasha et Kao, 1970). Chez les arbres fruitiers de la famille des Prunus, elle a été expérimentée depuis plus d'un demi-siècle (Tukey, 1934) et est devenue une procédure de routine dans la sélection de variétés de pêches à maturité très précoce (Monet, 1968). Elle a également été utilisée avec succès chez d'autres espèces fruitières comme l'avocat (Skene et Barlass, 1983) ou la banane (Cox et al., 1960).

Dans le cas où la culture in vitro d'embryons de très petite taille se montre difficile, voire impossible, la culture in vitro d'ovules fécondés permet dans certains cas de pallier cette difficulté. Cette technique de la culture d'embryons in ovulo a été appliquée récemment chez le pêcher (Toledo et al., 1980; Ramming, 1985). Son application à la vigne ne date également que de quelques années (Cain et al., 1983; Emershad et Ramming, 1984; Spiegel-Roy et al., 1985; Goldy et Amborn, 1987; Gray et al., 1987; Barlass et al., 1988).

En 1987, un programme de création variétale basé sur cette technique a été lancé à la station de recherches viticoles de Montpellier (France), en collaboration avec la division de recherches en horticulture du CSIRO de Merbein (Australie), qui a détaché à cet effet un de ses chercheurs en France pour une période de 6 mois. Ce programme $a$, en outre, bénéficié d'un appui financier de la part de l'Office National Interprofessionnel des Fruits et Légumes et de l'Horticulture (ONIFLHOR), dans le cadre du plan de relance du raisin de table en France.

\section{Matériel et Méthodes}

Pour servir de base à cette expérimentaticn, 21 croisements différents ont été réalisés en juin 1987 dans les 2 domaines expérimentaux de la station de recherches viticoles de Montpellier (Tableau I).

Au domaine de Vassal, 9 croisements, numérotés $V_{1}$ à $V_{9}$, ont fait intervenir 10 variétés apyrènes INRA en cours de sélection et d'expérimentation. Ces variétés étaient issues de 5 croisements classiques de première génération : variété à pépins $x$ variété sans pépins (Tableau II). Au total, 71 inflorescences ont été castrées et pollinisées, avec un taux de réussite élevé, puisque 9087 baies ont pu être récoltées, soit en moyenne 128 baies par inflorescence.

Au domaine du Chapitre, 12 croisements, numérotés $C_{1}$ à $C_{12}$, ont fait intervenir 5 variétés apyrènes, dont 4 d'origine étrangère et une sélection INRA, ainsi que 2 variétés à pépins, dont l'une, l'Alphonse Lavallée, a été utilisée uniquement comme parent mâle, et 
Tableau I. Croisements réalisés, nombre de grappes pollinisées et nombre de baies récoltées pour les expériences de sauvetage d'embryons.

\begin{tabular}{|c|c|c|c|c|c|}
\hline $\begin{array}{l}\text { Numéro } \\
\text { du } \\
\text { croisement }\end{array}$ & Parent femelle & & Parent mâle & $\begin{array}{c}\text { Nombre de } \\
\text { grappes } \\
\text { pollinisées }\end{array}$ & $\begin{array}{c}\text { Nombre de } \\
\text { baies } \\
\text { récoltées }\end{array}$ \\
\hline V 1 & INRA Mpt 2121-61 & $x$ & INRA Mpt 1992-3 & 6 & 443 \\
\hline v 2 & INRA Mpt 1992-3 & $\mathrm{x}$ & INRA Mpt 2004-9 & 9 & 1870 \\
\hline V 3 & INRA Mpt 2004-9 & $x$ & INRA Mpt 2121-61 & 6 & 426 \\
\hline V 4 & INRA Mpt 2004-9 & $x$ & INRA Mpt 1992-3 & 5 & 421 \\
\hline V 5 & INRA Mpt 2223-27 & $x$ & INRA Mpt 2121-30 & 8 & 1598 \\
\hline V 6 & INRA Mpt 2223-60 & $\mathrm{x}$ & INRA Mpt 2121-30 & 8 & 1152 \\
\hline V7 & INRA Mpt 2223-8 & $x$ & INRA Mpt 2121-30 & 9 & 1176 \\
\hline V 8 & INRA Mpt 2212-5 & $\mathrm{x}$ & INRA Mpt 1993-15 & 10 & 1073 \\
\hline$\vee 9$ & INRA Mpt 2212-17 & $x$ & INRA Mpt 1993-15 & 10 & 928 \\
\hline \multicolumn{4}{|c|}{ Sous-total V (Domaine de Vassal) } & 71 & 9087 \\
\hline C 1 & INRA Bx 8538 & $\mathrm{x}$ & Cardinal & 11 & 500 \\
\hline $\mathrm{C}_{2}$ & INRA Bx 8538 & $x$ & Alphonse Lavallée & 4 & 343 \\
\hline C 3 & Perlette & $x$ & Cardinal & 5 & 304 \\
\hline C 4 & Perlette & $x$ & Alphonse Lavallée & 2 & 206 \\
\hline C 5 & Sultanine & $x$ & Cardinal & 5 & 291 \\
\hline C 6 & Sultanine & $x$ & Alphonse Lavallée & 3 & 0 \\
\hline C 7 & Sultana Moscata & $x$ & INRA Bx 8538 & 2 & 237 \\
\hline $\mathrm{C} 8$ & Canner Seedless & $x$ & Cardinal & 8 & 250 \\
\hline $\mathrm{cg}$ & Canner Seedless & $\mathrm{x}$ & INRA Bx 8538 & 5 & 86 \\
\hline C 10 & Cardinal & $x$ & Perlette & 5 & 73 \\
\hline $\mathrm{C}_{11}$ & Cardinal & $x$ & Sultanine & 3 & 67 \\
\hline C 12 & Cardinal & $x$ & INRA Bx 8538 & 6 & 54 \\
\hline \multicolumn{4}{|c|}{ Sous-total C (Domaine du Chapitre) } & 59 & 2411 \\
\hline \multicolumn{4}{|l|}{ Total } & 130 & 11498 \\
\hline
\end{tabular}

Tableau II. Variétés de raisins de table sans pépins utilisées dans les croisements réalisés à Montpellier en 1987 (sélections INRA et variétés étrangères).

$\begin{array}{ll}\text { INRA Mpt 2004-9 } & \text { B } \\ \text { INRA Mpt 2121-30 } & \text { N } \\ \text { INRA Mpt 2121-61 } & \text { N } \\ \text { INRA Mpt 2212-5 } & \text { N } \\ \text { INRA Mpt 2212-17 } & \text { N } \\ \text { INRA Mpt 1993-15 } & \text { B } \\ \text { INRA Mpt 1992-3 } & \text { B } \\ \text { INRA Mpt 2223-8 } & \text { B } \\ \text { INRA Mpt 2223-27 } & \text { B } \\ \text { INRA Mpt 2223-60 } & \text { B } \\ \text { INRA Bx 8538 } & \text { B }\end{array}$

Sultanine blanche

Perlette B

Sultana moscata

Canner seedless

\section{B}

B

$\begin{array}{cc}\text { Cardinal } & \mathrm{x} \\ \text { Alphonse Lavallée } & \mathrm{x} \\ \text { id. } & \mathrm{x} \\ \text { Alphonse Lavallée } & \mathrm{x} \\ \text { id. } & \mathrm{x} \\ \text { Dattier de Beyrouth } & \mathrm{x} \\ \text { Dattier de Beyrouth } & \mathrm{x} \\ \text { id. } & \mathrm{x} \\ \text { id. } & \mathrm{x} \\ \text { id. } & \mathrm{x} \\ \text { Italia } & \mathrm{x}\end{array}$

Sultanine blanche

Sultanine blanche

id.

Sultana moscata

id.

Sultanine blanche

Sultana moscata

id.

id.

id.

Beauty seedless
Sultanine blanche Sultanine blanche Sultanine blanche 
l'autre, le Cardinal, a été utilisée simultanément comme parent mâle et parent femelle dans des croisements réciproques avec les variétés apyrènes. Le Cardinal, variété très précoce, est en effet connu pour le faible taux de germination de ses pépins. Au total, 59 inflorescences ont été castrées et pollinisées, avec un taux de réussite sensiblement plus faible qu'au domaine de Vassal, puisque seulement 2411 baies ont pu être récoltées, soit en moyenne 41 baies par inflorescence.

Les baies ont été récoltées à 3 dates différentes : le 20 juillet, soit un mois après la fin des pollinisations, le 3 août et le 17 août. Après récolte, les baies ont été stockées au froid $\left(+5^{\circ} \mathrm{C}\right)$ et à l'obscurité, pendant des durées variables, jusqu'à l'extraction et la mise en culture des ovules fécondés. La désinfection des baies a consisté en un trempage dans l'alcool à $96^{\circ}$ pendant une minute, suivi d'un flambage rapide. Les ovules, extraits aseptiquement après dissection des baies, ont été mis en culture dans des boites de Petri de $55 \mathrm{~mm}$ de diamètre (5 ovules par boîte), sur un milieu de Murashige et Skoog (1962) modifié, constitué d'un milieu de base (milieu $\mathrm{MB}$ ou $\mathrm{E}_{0}$ ), dont la composition est donnée sur le Tableau III, additionné de $10^{-5} \mathrm{M}$ d'acide indolyl-acétique (AIA) et $10^{-6} \mathrm{M}$ d'acide gibbérellique $\left(\mathrm{GA}_{3}\right)$, selon Spiegel-Roy et al. (1985). Les substances de croissance ont été ajoutées au milieu de culture après stérilisation de celui-ci à l'autoclave pendant $30 \mathrm{~min}$ à $115^{\circ} \mathrm{C}$. Le milieu de culture des ovules, appelé $E_{1}$, a été additionné ou non de charbon végétal activé (Prolabo) à la concentration de $0,25 \%$.

Après la mise en culture des ovules, les boîtes de Petri ont été placées dans une armoire climatique régulée à $25-28{ }^{\circ} \mathrm{C}$, soit en obscurité complète, soit en

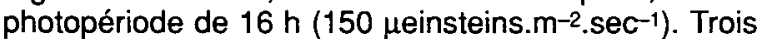
mois après les premières mises en culture d'ovules,

Tableau III. Composition du milieu de base $\left(E_{0}\right)$.

Macroéléments de Murashige et Skoog dilués $1 / 2$

Microéléments de Murashige et Skoog

$\begin{array}{lr}\text { Fer-EDTA } \begin{array}{lr}\mathrm{Fe} \mathrm{SO} \\ \mathrm{Na}_{2} \text { EDTA }\end{array} & 27,8 \mathrm{mg} / \mathrm{H} \\ & 37,2 \mathrm{mg} / \mathrm{l} \\ \text { Vitamines } & \\ \text { Mesoinositol } & 100 \mathrm{mg} / \mathrm{l} \\ \text { Acide nicotinique } & 10 \mathrm{mg} / \mathrm{l} \\ \text { Thiamine } \mathrm{HCl} & 10 \mathrm{mg} / \mathrm{l} \\ \text { Pyridoxine } \mathrm{HCl} & 1 \mathrm{mg} / \mathrm{l} \\ \text { Panthoténate de calcium } & 1 \mathrm{mg} / \mathrm{l} \\ \text { Biotine } & 0,01 \mathrm{mg} / \mathrm{l}\end{array}$

Acides aminés

Hydrolysat de caséine $\quad 100 \mathrm{mg} / \mathrm{l}$

Glutamine $\quad 100 \mathrm{mg} / \mathrm{l}$

Cystéine $\quad 100 \mathrm{mg} / \mathrm{l}$

Phénylalanine $10 \mathrm{mg} / \mathrm{l}$

Glycocolle $2 \mathrm{mg} / \mathrm{l}$

Saccharose

$20 \mathrm{mg} / \mathrm{l}$

Agar-Agar (Bacto-Difco)

$8 \mathrm{~g} / \mathrm{l}$ ceux-ci ont été disséqués sous une loupe binoculaire et les embryons vivants identifiables, du stade globulaire (Fig. 2) au stade torpille, extraits et mis en culture dans des boîtes de Petri contenant le même milieu de base que pour les cultures d'ovules, mais sans charbon actif et avec différentes compositions en substances de croissance :

$$
\begin{gathered}
E_{0}=\text { milieu de base }(M B) \text { sans substance } \\
\text { de croissance } \\
E_{1}=M B+A I A\left(10^{-5} M\right)+G_{3}\left(10^{-6} M\right) \\
E_{2}=M B+\text { benzyl-aminopurine (BAP à 10-6 M) } \\
E_{3}=M B+A I A\left(10^{-5} M\right)+G_{3}\left(10^{-6} M\right)+B A P\left(10^{-6} M\right)
\end{gathered}
$$

Au bout d'un mois de culture en photopériode de $16 \mathrm{~h}$, les embryons ayant développé une ébauche de feuille ont été transférés dans des tubes contenant le milieu $E_{0}$ sans substance de croissance et sans charbon actif, afin de parfaire leur développement (Fig. 3). Avant d'être acclimatées, les plantules racinées ainsi

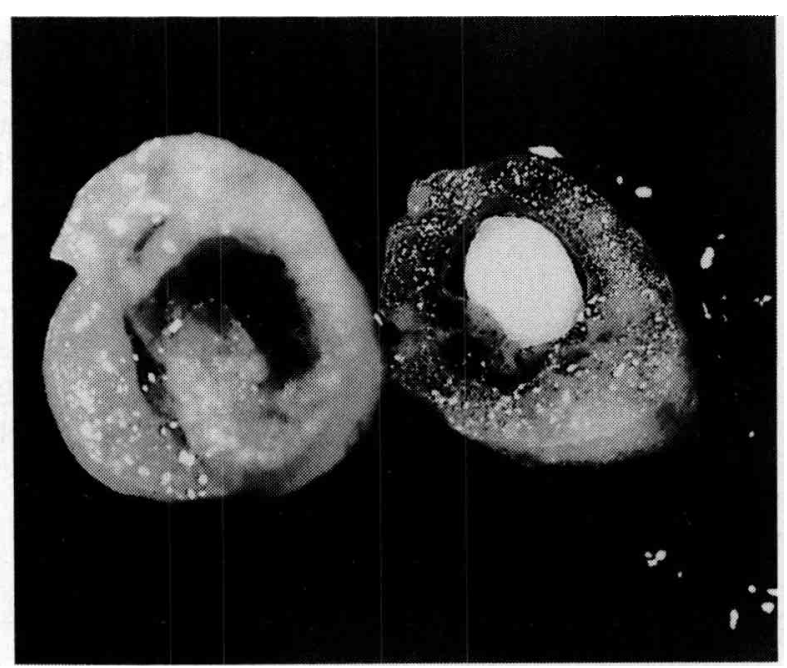

Fig. 2 Embryon globulaire dans une ébauche de pépin de la variété Sultana moscata cultivée in vitro (grossissement : $x 20$ ).

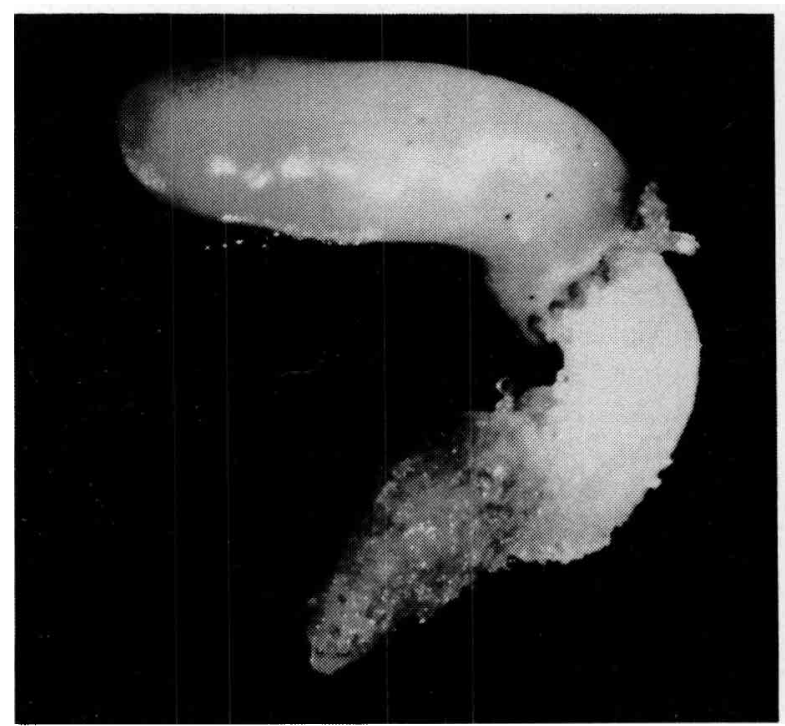

Fig. 3. Ebauche de pépin de la variété INRA Mpt 1992-3 germant in vitro (grossissement : $\times 20$ ). 
obtenues ont été multipliées in vitro, par microbouturage sur un milieu de Knop modifié (Galzy, 1964). Après extraction des tubes de culture, les plantes ont été repiquées dans des godets de tourbe remplis de perlite et maintenues en chambre climatisée pendant 15 jours sous des mini-serres en plastique, puis transplantées en serre dans des pots de 0,5 I remplis d'un milieu sable-tourbe (1:1). Après 6 mois de croissance en serre, les plantes ont été transférées au vignoble, afin d'y être soumises le plus rapidement possible à une sélection sévère portant sur le caractère apyrène, la taille des baies et la précocité de maturation.

\section{Résultats et Discussion}

L'ensemble des résultats obtenus est présenté dans le Tableau IV, qui sépare le Cardinal (variété à pépins) des variétés apyrènes. On peut cependant noter que le résultat final obtenu sur cette variété (nombre de plantes pour 100 ovules mis en culture) est pratiquement identique à celui obtenu avec l'ensemble des variétés apyrènes. Le taux d'embryogenèse (pourcentage d'ovules présentant un ou plusieurs embryons) plus élevé est compensé par un taux de développement des embryons en plantes légèrement plus faible et surtout par une perte beaucoup plus importante d'ovules, du fait de contaminations par des micro-organismes au moment de la mise en culture. En effet, celle-ci a été considérablement gênée, pour les baies prélevées au mois d'août, par leur état de maturité avancée, le Cardinal étant une des variétés de raisins de table les plus précoces.

On peut également remarquer que le nombre moyen d'ovules mis en culture par baie récoltée est inférieur à l'unité chez le Cardinal $(0,73)$, alors qu'il est pratiquement le double chez les variétés apyrènes $(1,36)$. Cette discordance doit être mise en relation avec le faible taux de succès enregistré dans les croisements faisant inter- venir le Cardinal comme parent femelle. De nombreuses baies obtenues étaient en fait millérandées, c'est-à-dire d'origine parthénocarpique.

II faut également souligner que le nombre d'embryons extraits et le nombre de plantes obtenues, présentés dans le Tableau IV, sont en fait inférieurs aux nombres réellement observés. En effet, chez un pourcentage non négligeable d'ovules (de 5 à 10\% selon les descendances), on a pu noter la présence d'embryons multiples, le nombre de ceux-ci variant de 2 à 19 pour un ovule issu de la variété Perlette. L'origine de ces embryons multiples est encore obscure. S'agit-il d'un phénomène de polyembryonie nucellaire analogue à ce qui est observé classiquement chez les Citrus (Maheshwari, 1950)? La polyembryonie est un phénomène rare chez la vigne (Bouquet, 1980). Elle peut cependant être favorisée par des conditions de culture in vitro. II est possible, sinon probable, qu'il s'agisse d'embryons somatiques adventifs qui se sont développés à partir de l'embryon zygotique. L'embryogenèse somatique adventive en culture in vitro a été observée chez la vigne par Krul et Worley (1977) et de nombreux auteurs. Récemment, Stamp et Meredith (1988) ont induit la prolifération d'embryons somatiques à partir d'embryons zygotiques cultivés in vitro. Seules l'observation et la comparaison au vignoble des plantes issues de ces embryons multiples permettront d'avoir quelques éléments de réponse à cette question. Quoi qu'il en soit, les embryons surnuméraires et les plantes qui en sont issues n'ont pas été pris en compte dans les résultats, de manière à ne pas fausser les pourcentages. Un embryon multiple a donc été considéré comme embryon unique, même s'il a pu donner naissance à plusieurs plantes. De même, celles-ci, bien qu'elles aient été individualisées pour pouvoir être comparées entre elles au vignoble, ont été comptabi-

Tableau IV. Résultats des cultures in vitro d'ovules et d'embryons, effectuées en 1987-1988 à l'INRA de Montpellier.

Ensemble Cardinal Total
apyrènes

\footnotetext{
Nombre de baies récoltées

Nombre d'ovules mis en culture

Nombre d'ovules perdus (contaminés)

Nombre d'ovules disséqués

Nombre d'embryons extraits

Pourcentage embryogenèse

Nombre d'embryons perdus (contaminés)

Nombre d'embryons développés en plantes

Pourcentage développement

obtenues pour 100

ovules cultivés
}

11304

15467

1439

14028

3408

24,3

238

1254

39,6

8,1

194
143
41
102
37
36,3
4
12
36,4
8,4

11498

15610

1480

14130

3445

24,4

242

1266

39,4 
lisées comme plante unique. Au total, il faut donc ajouter 120 plantes supplémentaires, qui ne sont probablement pas des génotypes originaux, aux 1266 plantes indiquées dans le Tableau IV.

Si on compare (Tableau $\mathrm{V}$ ) les résultats obtenus à Montpellier en 1987-1988 avec ceux obtenus par les chercheurs de l'USDA à Fresno (Californie) de 1983 à 1986, ainsi qu'avec ceux obtenus par les chercheurs du CSIRO à Merbein (Australie) en 1988, on constate une similitude frappante des chiffres, alors que ces résultats ont été obtenus dans des milieux très différents, avec des variétés différentes et en utilisant des méthodologies sensiblement différentes.

L'analyse de variance appliquée aux pourcentages d'embryons extraits des ovules (après transformation angulaire) fait ressortir un certain nombre de points intéressants (Tableau VI).

1. L'influence de la variété sur le taux d'obtention des embryons est hautement significative. En revanche, la variété ne semble pas avoir d'effet sur le taux de développement de ces embryons en plantes (Tableau VII). Le test des rangs multiples de Duncan permet de classer les variétés étudiées en 3 groupes :

- les variétés à faible taux d'embryogenèse (2 à $7 \%$ ) : c'est le cas de la Sultanine blanche, de la sélection INRA Mpt 2004-9 et de la Perlette, qui se distingue toutefois des 2 précédentes;

- les variétés à taux moyen d'embryogenèse (12 à 15\%) : c'est le cas du Canner seedless et des 3 sélections INRA, Mpt 1992-3, Mpt 2121-61 et Bx 8538;

- les variétés à taux élevé d'embryogenèse $(30 \%$ et plus) : c'est le cas de la Sultana moscata et des autres sélections INRA, qui sont d'ailleurs comparables au Cardinal pour cette caractéristique.

$\mathrm{Si}$, pour une variété donnée, on a pu observer une étroite corrélation entre la grosseur des ébauches de pépins et la présence ou non d'embryons viables, il n'en est pas de même entre variétés, comme le montre le Tableau VII.

Tableau V. Comparaison des résultats de cultures in vitro d'ovules et d'embryons effectuées à Montpellier (France), Fresno (Etats-Unis) et Merbein (Australie).

\begin{tabular}{lcccccc}
\hline & $\begin{array}{c}\text { Nombre } \\
\text { d'ovules } \\
\text { cultivés }\end{array}$ & $\begin{array}{c}\text { Nombre } \\
\text { d'embryons } \\
\text { extraits }\end{array}$ & Pourcentage & $\begin{array}{c}\text { Nombre } \\
\text { de plantes } \\
\text { obtenues }\end{array}$ & $\begin{array}{c}\text { Pourcentage } \\
\text { (1) }\end{array}$ & $\begin{array}{c}\text { Pourcentage } \\
\text { (2) }\end{array}$ \\
\hline Montpellier (1987-1988) & 15610 & 3445 & 22,1 & 1266 & 36,7 & 8,1 \\
Fresno* (1983-1986) $_{\text {Merbein }}^{\star \star}(1988)$ & 37360 & 8775 & 23,4 & 2718 & 31,0 & 7,2 \\
& 6933 & 1148 & 16,6 & 399 & 34,8 & 5,8
\end{tabular}

(1) Par rapport aux embryons extraits; (2) Par rapport aux ovules cultivés; " d'après Barlass et al., 1988; "* Davis (communication personnelle).

Tableau VI. Analyse de variance appliquée aux pourcentages d'embryons extraits des ovules (après transformation angulaire).

\begin{tabular}{|c|c|c|c|c|c|}
\hline Variation & $\begin{array}{l}\text { Degrés de } \\
\text { liberté }\end{array}$ & $\begin{array}{c}\text { Somme des carrés } \\
\text { des écarts }\end{array}$ & $\begin{array}{l}\text { Carrés } \\
\text { moyens }\end{array}$ & $F$ & Prob $>F$ \\
\hline Totale & 155 & 8,8442 & & & \\
\hline Variétés & 12 & 4,6365 & 0,3864 & 42,87 & 0,0001 \\
\hline Date & 2 & 1,6171 & 0,8085 & 89,71 & 0,0001 \\
\hline Charbon & 1 & 0,5572 & 0,5572 & 61,83 & 0,0001 \\
\hline Photopériode & 1 & 0,0015 & 0,0015 & 0,17 & 0,6849 \\
\hline Var. $x$ date & 24 & 1,0403 & 0,0433 & 4,81 & 0,0001 \\
\hline Var. $x$ charbon & 12 & 0,1388 & 0,0116 & 1,28 & 0,2431 \\
\hline Var. x photop. & 12 & 0,0454 & 0,0037 & 0,42 & 0,9519 \\
\hline Date $x$ charbon & 2 & 0,0066 & 0,0033 & 0,37 & 0,6946 \\
\hline Date $x$ photop. & 2 & 0,0057 & 0,0028 & 0,32 & 0,7300 \\
\hline Charbon $x$ photop. & 1 & 0,0199 & 0,0199 & 2,22 & 0,1403 \\
\hline Erreur & 86 & 0,7751 & 0,0090 & & \\
\hline
\end{tabular}


Ainsi, la variété Canner seedless et les 3 sélections INRA, Mpt 1992-3, Mpt 2121-61 et Bx 8538 , présentent pratiquement le même taux d'embryogenèse, alors que la dimension et le degré de lignification de leurs ébauches de pépins, mesurés par le poids moyen sec de celles-ci, sont très différents. De même, la variété Sultana moscata et les autres sélections INRA présentent des taux d'embryogenèse élevés et très voisins, alors que leurs ébauches de pépins sont également très différentes.

2. L'influence de la date de récolte des baies sur le taux d'obtention des embryons est hautement significative. Les ovules prélevés sur des baies récoltées tardivement, au voisinage de la véraison, présentent les plus forts taux d'embryogenèse (Tableau VIII). On peut cependant noter une interaction significative entre la variété et la date de récolte. L'influence de cette dernière sur le taux d'obtention des embryons semble moins nette avec les variétés à faible taux d'embryogenèse (Sultanine, Perlette, INRA Mpt 2004-9) qu'avec les autres. Cependant, Spiegel-Roy et al. (1985) ont également observé un effet favorable des prélèvements tardifs sur l'obtention de plantes à partir d'ovules fécondés de la variété Perlette.

En revanche, la date de récolte des baies n'a aucun effet sur le taux de développement des embryons en plantes, du moins chez les variétés

Tableau VII. Influence de la variété sur le taux d'obtention d'embryons en culture d'ovules et sur le pourcentage de développement de ceux-ci en plantes entières.

\begin{tabular}{lcccc}
\hline Variété & $\begin{array}{c}\text { Nombre } \\
\text { d'ovules } \\
\text { cultivés }\end{array}$ & $\begin{array}{c}\text { Poids } \\
\text { moyen sec } \\
\text { des ovules }(\mathrm{mg})\end{array}$ & $\begin{array}{c}\text { Pourcentage } \\
\text { d'embryons }^{\text {obtenus }}\end{array}$ & $\begin{array}{c}\text { Pourcentage } \\
\text { de plantes } \\
\text { developpées }\end{array}$ \\
\hline Sultanine blanche & 205 & 1,0 & 2,0 & 50,0 \\
Perlette & 207 & 1,5 & 6,8 & 71,4 \\
INRA Mpt 2004-9 & 690 & 2,0 & 3,0 & 33,3 \\
Canner seedless & 300 & & 14,3 & 34,9 \\
INRA Mpt 1992-3 & 2199 & 2,2 & 13,5 & 24,7 \\
INRA Mpt 2121-61 & 757 & 2,6 & 12,3 & 31,5 \\
INRA Bx 8538 & 960 & 6,5 & 12,5 & 24,2 \\
Sultana moscata & 185 & 8,5 & 33,0 & 44,3 \\
INRA Mpt 2212-5 & 1482 & & 29,5 & 36,4 \\
INRA Mpt 2212-17 & 1435 & 2,5 & 32,5 & 41,1 \\
INRA Mpt 2223-27 & 2690 & 4,0 & 34,1 & 44,1 \\
INRA Mpt 2223-8 & 1150 & 5,4 & 34,6 & 25,9 \\
INRA Mpt 2223-60 & 1768 & 9,3 & 31,1 & 35,2 \\
Cardinal (non apyrène) & 102 & 16,2 & 36,3 & 32,4 \\
\hline
\end{tabular}

\footnotetext{
* Par rapport au nombre d'embryons obtenus.
}

Tableau VIII. Effet de la date de récolte des baies sur le taux d'obtention d'embryons en culture d'ovules et sur le pourcentage de développement de ceux-ci en plantes entières.

\begin{tabular}{rcccccc}
\hline $\begin{array}{c}\text { Date de } \\
\text { récolte }\end{array}$ & $\begin{array}{c}\text { Nombre } \\
\text { d'ovules } \\
\text { cultivés }\end{array}$ & $\begin{array}{c}\text { Nombre } \\
\text { d'embryons } \\
\text { extraits }\end{array}$ & Pourcentage & $\begin{array}{c}\text { Nombre } \\
\text { d'embryons } \\
\text { cultivés }{ }^{* *}\end{array}$ & $\begin{array}{c}\text { Nombre } \\
\text { de plantes } \\
\text { obtenues }\end{array}$ & Pourcentage \\
\hline $20 / 7$ & 5046 & 673 & 13,3 & 385 & 171 & 44,4 \\
$3 / 8$ & 4721 & 1035 & 21,9 & 580 & 252 & 43,4 \\
$17 / 8$ & 4261 & 1700 & 39,9 & 1059 & 477 & 45,0 \\
Total & 14028 & 3408 & 24,3 & 2024 & 900 & 44,5
\end{tabular}

\footnotetext{
"Ensemble des descendances; ** Descendances $V_{2}$ et $V_{5}$ a $V_{9}$ (embryons repiqués sur milieux $E_{0}$ et $E_{2}$ ).
} 
présentant un taux d'embryogenèse moyen ou élevé.

3. L'addition de charbon actif au milieu de culture des ovules augmente nettement le taux d'obtention des embryons, pour toutes les variétés et quelles que soient la date de récolte des baies et les conditions d'éclairement en culture in vitro (interactions non significatives). Quant à l'effet du charbon actif sur le taux de développement des embryons en plantes, il semble dépendre de la variété. Pratiquement nul pour les descendances $V_{6}$ à $V_{9}$, il est nettement favorable pour la descendance $V_{5}$ (Tableau IX). II faut noter que les embryons issus de cette descendance présentent le taux de développement en plantes le plus élevé $(44,1 \%)$.

4. Les conditions d'éclairement des cultures d'ovules n'ont aucun effet sur le taux d'obtention des embryons, celui-ci étant pratiquement identique dans les 2 cas : $24,2 \%$ à l'obscurité contre $24,4 \%$ avec photopériode, pour l'ensemble des variétés et des dates de récolte. II ne semble pas y avoir d'interaction entre l'éclairement des cultures et la présence ou non de charbon actif dans le milieu. Le taux de développement en plantes des embryons n'est également pas influencé par les conditions d'éclairement des cultures d'ovules.

5. L'influence de la conservation au froid des baies après récolte et avant extraction et mise en culture des ovules, sur le taux d'obtention des embryons, semble dépendre assez nettement des variétés utilisées (Tableau $X$ ). Pour les descendances $V_{5}$ et $V_{9}$, on peut observer un effet favorable, quelle que soit la date de récolte des baies. En revanche, pour les descendances $V_{5}$, $V_{7}$ et $V_{8}$, l'effet du froid semble dépendre de la date de récolte des baies : favorable pour les baies récoltées précocement, il devient défavorable pour les baies récoltées plus tardivement. II faut toutefois noter que la durée de conservation au froid des baies n'a pas été identique pour les

Tableau IX. Effet de la présence de charbon actif $(0,25 \%)$ dans le milieu de culture d'ovules sur le taux d'obtention d'embryons et le pourcentage de développement de ceux-ci en plantes entières.

\begin{tabular}{|c|c|c|c|c|c|c|c|}
\hline Milieu & $\begin{array}{c}\text { Nombre } \\
\text { d'ovules } \\
\text { cultivés } \\
\left({ }^{*}\right)\end{array}$ & $\begin{array}{c}\text { Nombre } \\
\text { d'embryons } \\
\text { extraits }\end{array}$ & Pourcentage & $\begin{array}{c}\text { Nombre } \\
\text { d'embryons } \\
\text { cultivés } \\
\left(^{* *}\right)\end{array}$ & $\begin{array}{c}\text { Pourcentage } \\
\text { de plantes } \\
\text { obtenues }\end{array}$ & $\begin{array}{c}\text { Nombre } \\
\text { d'embryons } \\
\text { cultivés } \\
\left(^{* * *}\right)\end{array}$ & $\begin{array}{c}\text { Pourcentage } \\
\text { de plantes } \\
\text { obtenues }\end{array}$ \\
\hline $\begin{array}{l}\text { Témoin } \\
\text { Charbon }\end{array}$ & $\begin{array}{l}7482 \\
6546\end{array}$ & $\begin{array}{l}1482 \\
1926\end{array}$ & $\begin{array}{l}19,8 \\
29,4\end{array}$ & $\begin{array}{l}313 \\
321\end{array}$ & $\begin{array}{l}47,9 \\
61,4\end{array}$ & $\begin{array}{l}591 \\
640\end{array}$ & $\begin{array}{l}37,4 \\
38,4\end{array}$ \\
\hline Total & 14028 & 3408 & 24,3 & 634 & 54,7 & 1231 & 37,9 \\
\hline
\end{tabular}

Tableau X. Effet de la date de récolte des baies et de la conservation au froid de celles-ci sur le taux d'obtention d'embryons en culture d'ovules.

\begin{tabular}{|c|c|c|c|c|c|c|}
\hline \multirow[b]{2}{*}{ Date de récolte des baies } & \multicolumn{3}{|c|}{ Descendances $V_{2}, V_{6}, V_{7}, V_{8}$} & \multicolumn{3}{|c|}{ Descendances $V_{5}$ et $V_{9}$} \\
\hline & $20 / 7$ & $3 / 8$ & $17 / 8$ & $20 / 7$ & $3 / 8$ & $17 / 8$ \\
\hline \multicolumn{7}{|l|}{ Sans conservation au froid } \\
\hline Nombre d'ovules cultivés & 850 & 815 & 880 & 380 & 395 & 415 \\
\hline Nombre d'embryons obtenus & 123 & 195 & 362 & 33 & 114 & 186 \\
\hline Pourcentage & 14,5 & 23,9 & 41,1 & 8,7 & 28,9 & 44,8 \\
\hline \multicolumn{7}{|l|}{ Avec conservation au froid } \\
\hline Durée moyenne de conservation & $45 \mathrm{j}$ & $(10-22 j)$ & $15 \mathrm{j}$ & $45 j$ & $(10-22 j)$ & $15 j$ \\
\hline Nombre d'ovules cultivés & 1169 & 1710 & 1175 & 1140 & 780 & 1015 \\
\hline Nombre d'embryons obtenus & 231 & 371 & 398 & 172 & 257 & 611 \\
\hline Pourcentage & 19,8 & 21,7 & 33,8 & 15,1 & 32,9 & 60,2 \\
\hline
\end{tabular}


3 dates de récolte. D'autre part, on peut également noter que les descendances $V_{5}$ et $V_{9}$ présentent en moyenne les taux de développement des embryons en plantes les plus élevés $(44,1$ et $41,1 \%)$.

6. Comme on pouvait s'y attendre, la composition du milieu de culture des embryons influe considérablement sur le taux de développement de ceux-ci en plantes entières (Tableau XI). Sur l'ensemble des variétés, le milieu le plus favorable est le milieu de base sans substance de croissance (milieu $E_{0}$ ). Le milieu de culture des ovules (milieu $E_{1}$ ) ne semble pas être favorable au bon développement des embryons, bien qu'un certain nombre d'ovules aient été capables de germer naturellement sur ce milieu, avant leur dissection (Fig. 4). L'addition de benzylaminopurine (milieux $E_{2}$ et $E_{3}$ ) diminue sensiblement les taux de développement en plantes. On sait qu'une culture de courte durée sur milieu additionné de BAP améliore le taux de "conversion" (Redenbaugh, 1988) des embryons somatiques de vigne (Krul, 1985; Bouquet et al., 1987). II ne semble pas en être de même pour les embryons zygotiques, qui ne présentent d'autre part aucune manifestation d'un quelconque phénomène de dormance. L'absence de dormance des embryons somatiques obtenus à partir de variétés apyrènes a également été observée (Benchetrit, 1987), confirmant les observations de Bouquet et al. (1987) sur l'inefficacité des traitements au froid ou à l'acide gibbérellique pour améliorer les taux de "conversion» des embryons somatiques des cépages de Vitis vinifera $L$.

\section{Conclusions}

Les travaux de culture d'ovules et d'embryons in vitro, menés en 1987-1988 à la station de recherches viticoles de Montpellier, ont porté sur un nombre important d'ovules et de variétés. Ils

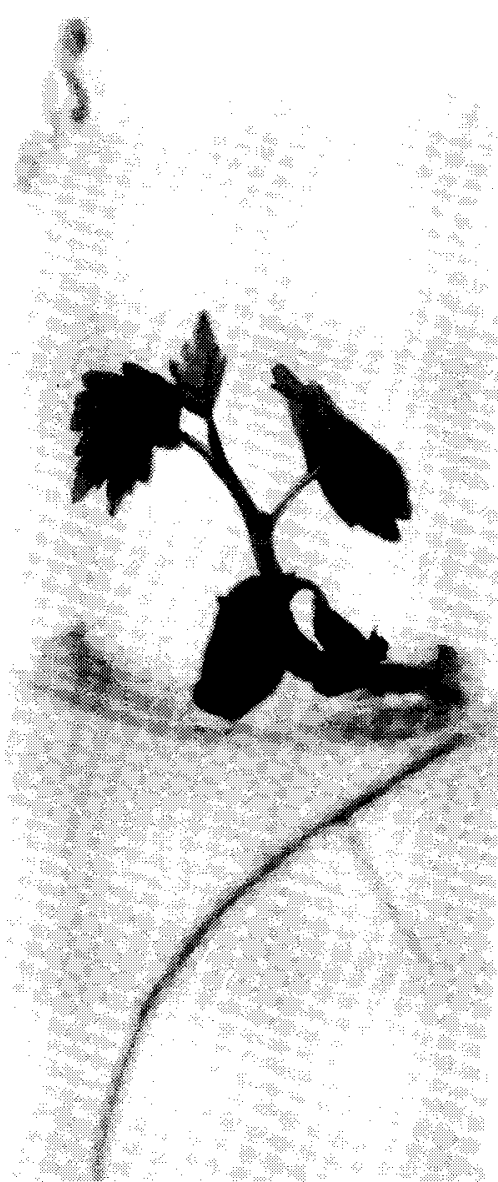

Fig. 4. Embryon de la variété Sultanine blanche se développant en plantule.

ont permis d'affiner la méthodologie d'une technique qui est désormais à la base de tous les programmes d'amélioration du raisin de table sans pépins, développés en France et à l'étranger.

$\mathrm{Au}$ vu des résultats obtenus, la réussite des cultures semble essentiellement conditionnée par le génotype de la variété apyrène utilisée comme parent femelle, ainsi que par l'époque de récolte des baies. L'influence favorable de la pré-

Tableau XI. Effet de la composition du milieu de culture des embryons sur leur taux de développement en plantes entières.

\begin{tabular}{lccr} 
Milieu de culture & $\begin{array}{c}\text { Nombre } \\
\text { d'embryons } \\
\text { cultivés }\end{array}$ & $\begin{array}{c}\text { Nombre } \\
\text { de plantes } \\
\text { obtenues }\end{array}$ & Pourcentage \\
\hline$E_{0}: M B$ & 909 & 507 & 55,8 \\
$E_{1}: M B+A I A+G_{3}$ & 421 & 118 & 28,0 \\
$E_{2}: M B+B A P$ & 1644 & 614 & 37,3 \\
$E_{3}: M B+$ AlA + GA $A_{3}+$ BAP & 229 & 27 & 11,3 \\
Total & 3203 & 1266 & 39,6
\end{tabular}


sence de charbon actif dans le milieu de culture doit être également soulignée. Cependant, la technique employée, exigeant plusieurs manipulations successives, est lourde à mettre en œuvre en routine sur de grands effectifs. Les améliorations à rechercher résident dans l'augmentation du taux de germination naturel des ovules cultivés in vitro, permettant ainsi d'éviter de disséquer ceux-ci afin d'en extraire les embryons. Mais cette germination se produit de manière erratique et les essais visant à mieux contrôler et homogénéiser celle-ci n'ont pas donné jusqu'à présent de résultats vraiment satisfaisants.

Quoi qu'il en soit, les centaines de plantes obtenues au cours de cet essai vont permettre de disposer d'une variabilité génétique considérable pour les travaux de sélection de nouvelles variétés de raisins de table sans pépins capables de satisfaire les exigences des viticulteurs et d'offrir aux consommateurs un produit nouveau et attractif.

\section{Références}

Barlass M., Ramming D.W. \& Davis H.P. (1988) In ovulo embryo culture : a breeding technique to rescue seedless $x$ seedless table grape crosses. Austr. Grapegrower Winemaker April, 123-125

Barritt B.H. (1970) Ovule development in seeded and seedless grapes. Vitis $9,7-14$

Benchetrit G. (1987) Influence de quelques facteurs sur la production de cals, d'embryons et de plantes entières par culture in vitro d'anthères de variétés de vigne apyrènes (Vitis vinifera L.). Mémoire DAA ENSAIA, Nancy

Bouquet A. (1980) Effect of some genetic and environmental factors on spontaneous polyembryony in grape (Vitis vinifera L.). Vitis 19, 134-150

Bouquet A., Fallot J., Lebrun L. \& Mauro M.C. (1987) Influence de quelques facteurs sur la production et le développement d'embryons somatiques obtenus par culture d'anthères in vitro chez Vitis vinifera L. In: Physiologie de la Vigne. CR $3^{e}$ Symposium International, Bordeaux, juin 1986, OIV, pp. 31-37

Cain D.W., Emershad R.L. \& Taralio R.E. (1983) In ovulo embryo culture and seedling development of seeded and seedless grapes. Vitis 22, 9-14

Cox E.A., Stotzky G. \& Goos R.D. (1960) In vitro culture of Musa balbisiana Colla embryos. Nature 185, 403404

Emershad R.L. \& Ramming D.W. (1984) In ovulo embryo culture of Vitis vinifera L. cv. Thompson seedless. Am. J. Bot. 71, 873-877

Galzy R. (1964) Technique de thermothérapie de la vigne. Ann. Epiphyt. (Paris) 15, 135-146

Goldy R.G. \& Amborn U. (1987) In vitro culturability of ovules from 10 seedless grape clones. Hortic. Science 22, 952

Gray D.J., Fisher L.C., Mortensen J.A., 1987. Comparison of methodologies for in ovulo embryo rescue of seedless grapes. Hort. Science 22, 1334
Hannig E. (1904) Zur Physiologie pflanzlicher Embryonen. Uber die Kultur von cruciferen Embryonen ausserhalb des Embryosacks. Bot. Zeit. 62, 45-80

Islam A.S. (1964) A rare hybrid combination through application of hormone and embryo culture. Nature 201,320

Kasha K.J. \& Kao K.N. (1970) High frequency haploid production in barley (Hordeum vulgare L.). Nature $225,874-875$

Krul W.R. (1985) Somatic embryo induction, expression and development : a general model. In: Amélioration de la Vigne et Culture in Vitro. (Moet-Hennessy Ed.), pp. 101-120

Krul W.R. \& Worley J.F. (1977) Formation of adventitious embryos in callus cultures of "Seyval», a French hybrid grape. J. Am. Soc. Hortic. Sci. 102, 360-363

Loomis N.H. \& Weinberger J.H. (1979) Inheritance studies of seedlessness in grapes. J. Am. Soc. Hortic. Sci. 104, 181-184

Maheshwari P. (1950) An Introduction to The Embryology of Angiosperms. McGraw Hill, New York

Mavrikios C. (1977) Problèmes sur l'emploi des gibberellines sur les raisins de table. Bull. OIV 50, 243-252

Murashige T. \& Skoog F. (1962) A revised medium for rapid growth and bioassays with tobacco tissue culture. Physiol. Plant. 15, 473-497

Monet R. (1968) Méthode permettant l'obtention de plantes viables à partir d'embryons de variétés très précoces chez le pêcher. Ann. Amelior. Plant. (Paris) $18,85-91$

Nitsch J.P., Pratt C., Nitsch C. \& Shaulis M.J. (1960) Natural growth substance in Concord and Concord seedless grapes in relation to berry development. $A m$. J. Bot. 47, 566-576

Ramming D.W. (1985) In ovulo embryo culture of early maturing Prunus. Hort. Science 20, 419-420

Redenbaugh K. (1988) Encapsulated plant embryos. In: Biotechnology in Agriculture. Adv. in Biotechnological Processes, vol. 9. (A. Mizrahi Ed.), pp. 225-248

Skene K.G.M. \& Barlass M. (1983) In vitro culture of abscised immature avocado embryos. Ann. Bot. 52, 667-672

Spiegel-Roy P., Sahar N., Baron J. \& Lavi U. (1985) In vitro culture and plant formation from grape cultivars with abortive ovules and seeds. J. Am. Soc. Hortic. Sci. 110, 109-112

Stamp J.A. \& Meredith C.P. (1988) Proliferative somatic embryogenesis from zygotic embryos of grapevine. J. Am. Soc. Hortic. Sci. 113, 941-945

Stewart J.M. \& Hsu C.L; (1978) Hybridization of diploid and tetraploid cottons through in ovulo embryo culture. J. Hered. 69, 404-408

Stout A.B. (1936) Breeding for hardy seedless grapes. Proc. Am. Soc. Hortic. Sci. 34, 416-420

Toledo W., Hugard J. \& Jonard R. (1980) Amélioration de la technique d'obtention de plants de semis de pêcher à partir de graines du cultivar de maturité précoce «Springtime». CR Acad. Sci. Paris 290, 539-542

Tukey H.B. (1934) Artificial culture methods for isolated embryos of deciduous fruits. Proc. Am. Soc. Hortic. Sci. 32, 313-322

Weinberger J.H. \& Harmon F.N. (1964) Seedlessness in vinifera grapes. Proc. Am. Soc. Hortic. Sci. 85, 270-274 